\title{
Anesthetic management of anaplastic thyroid carcinoma
}

Keywords: anaplastic thyroid carcinoma, preoperative workup, anterior neck pressure, retrosternal goitre, thoracic inlet

\section{Introduction}

Goitre are common, with current data suggesting a prevalence in North America as high as 7\% when assessed by palpation and $50 \%$ by ultrasonography or at autopsy. ${ }^{1}$ Many nodular goiters are asymptomatic, but larger goiters can compress the trachea and/ or esophagus, ${ }^{2}$ resulting in subjective symptoms such as shortness of breath, dysphagia, anterior neck pressure, and/or voice changes. Some patients may not notice compressive symptoms if their goiter has gradually increased in size. In a patient without any compressive complaints, obstruction may only be recognized on preoperative workup and imaging. ${ }^{3}$

Severe tracheal obstruction from massive thyroid disease that necessitates urgent airway intervention and sub sequent thyroid resection is well described in the literature. ${ }^{4-6}$

Retrosternal goitre is an uncommon problem encountered rarely by anaesthetists working outside specialized head and neck surgical units. While there are numerous reports in the literature concerning the surgical management of retrosternal goitre ${ }^{7-11}$ and ensuing problems ${ }^{11-13}$ those considering anaesthetic management are frequently limited to isolated case reports and small case series. ${ }^{13-15}$

Although retrosternal goitre is well described there is still much confusion as to the precise definition. In a review of 34 papers comprising 2426 patients, Huins and colleagues 5 found four definitions of retrosternal goitre. These definitions included the presence of a gland which:

a. Any part extends below the thoracic inlet with the patient in the surgical position,

b. More than $50 \%$ lies within the thoracic cavity,

c. Extends to the level of the fourth thoracic vertebra on chest $\mathrm{X}$-ray, and

d. Extends to the level of the aortic arch.

However, the majority of patients with large goiters do not present with impending respiratory collapse, and thyroid surgery can be performed electively. Thyroid goiters that cause tracheal compression or deviation theoretically pose potential challenges to intubation, but the most appropriate intubation approach in these cases remains controversial and based on medical specialty.

\section{Case report}

Female patient of 58 years of age, with a previous tumor of the neck of 30 years of evolution which presented greater increase in volume in 20 days, which was progressing, reaching a point at which food intake was hindered by the access to the mouth, which increased until dyspnea accompanied by fever, hyporexia, asthenia and adynamia, the patient was able to eat food in the form of mash. She avoided the conversation due to dyspnea, her limited ability to communicate due
Volume 10 Issue 5 - 2019

\section{Barbabosa Balanzario MA, Vázquez Roque $P$, Cortes Aceves HO, Puente Solorio A}

Department of Anesthesiology, Universidad Autónoma del Estado de México, Mexico

Correspondence: Barbabosa Balanzario MA, Anesthesiology Service of the Medical Center Lic, Adolfo López Mateos, Health Institute of the State of Mexico, Toluca, State of Mexico, Mexico, Tel 044722 5716794,Email manalebarbal@gmail.com

Received: September 22, 2019 | Published: October II, 2019

to dysphonia and the appearance of her face especially that of her neck, forced her to seek medical attention (Figure 1).

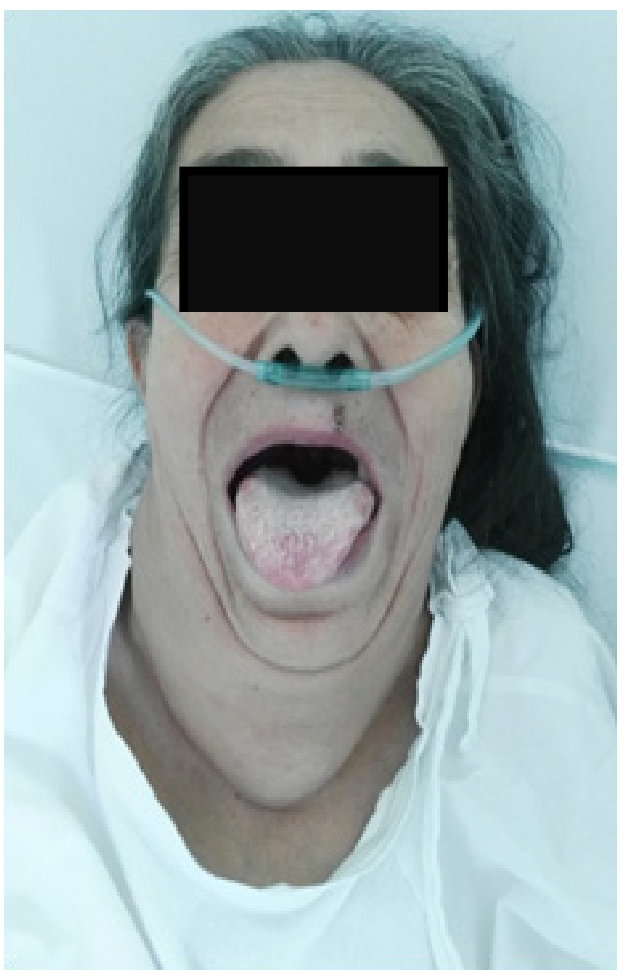

Figure I

Preoperative tests revealed no nutritional deficiencies. At the first physical examination, the patient is shown with a volume increase in the neck with a neck circumference of 66 centimeters, the largest limited oral opening, a chest $\mathrm{x}$-ray is performed presenting retrosternal goitre with displacement and narrowness of the airway to the left. Therefore, computed tomography is taken, revealing narrowness of the larynx of $7.4 \times 17.09 \mathrm{~mm}$ corroborated in the reconstruction of the airway (Figure 2-5), so it was scheduled by general surgery for a thyroidectomy plus tracheostomy. 


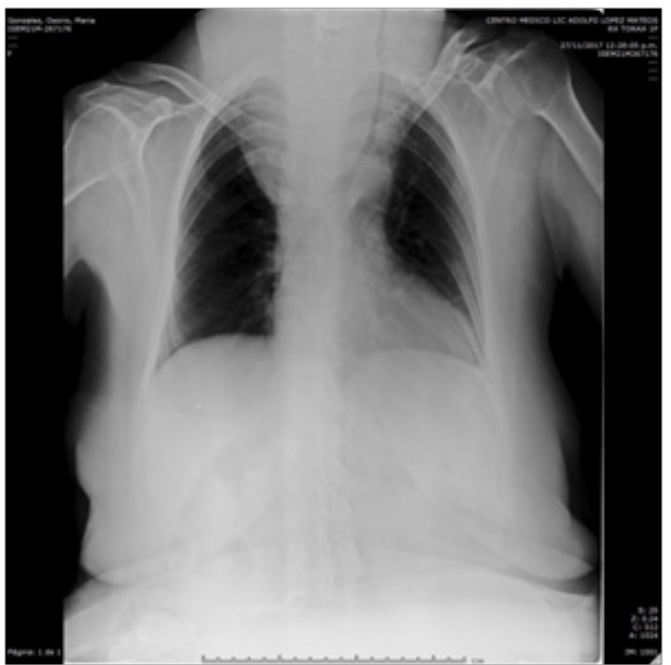

Figure 2 Imaging depicting tracheal deviation.

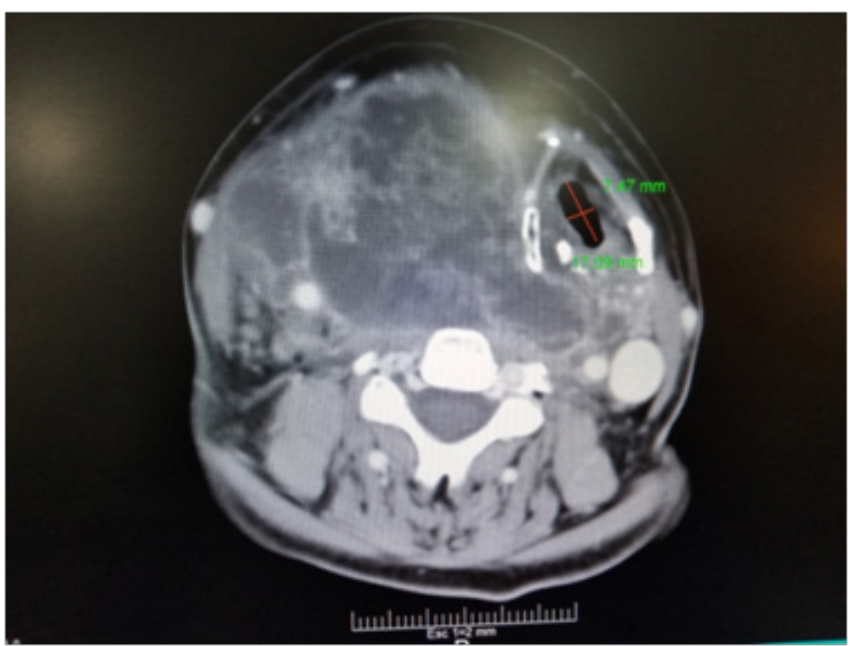

Figure 3 Imaging depicting tracheal deviation and compression.

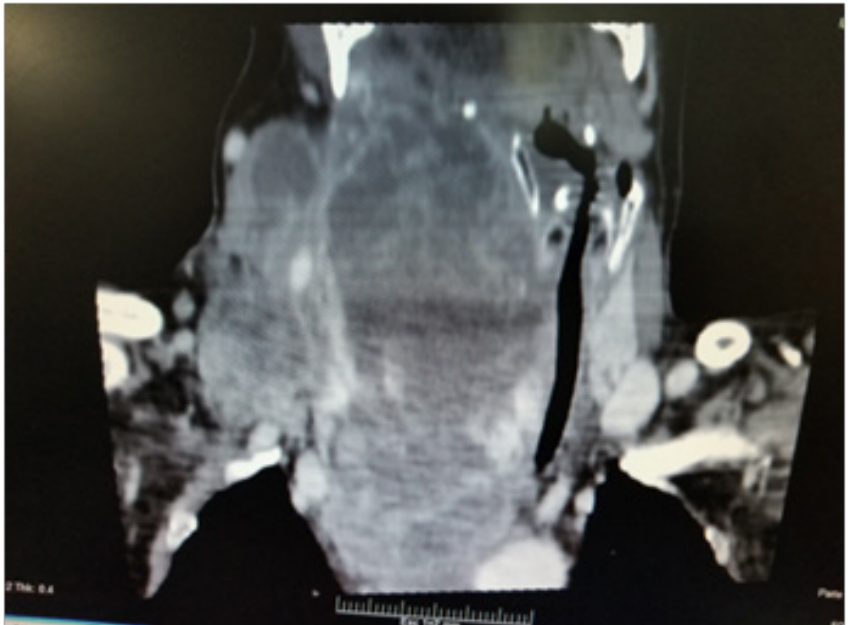

Figure 4 Imaging depicting tracheal deviation.

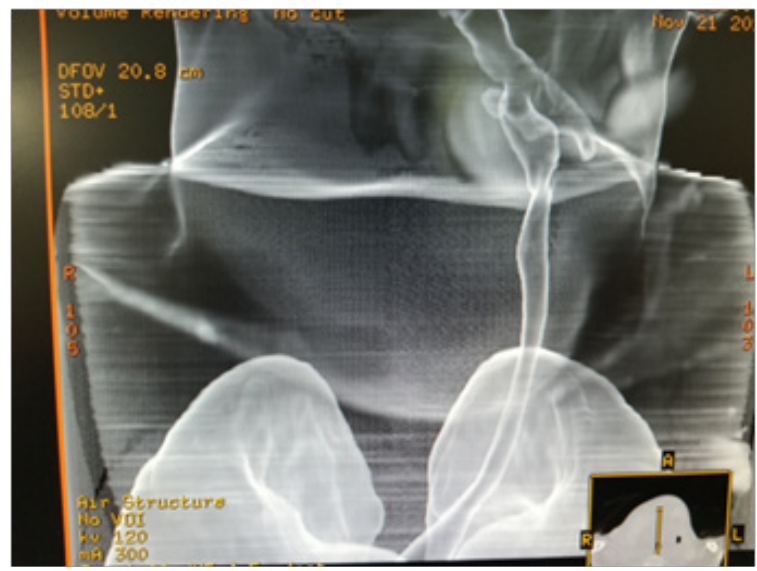

Figure $5 \mathrm{CT}$ scan with reconstruction of the airway.

He was given general anesthesia, with intubation in an awake patient, with flexible fibroscopy; the patient was placed in a sitting position, in the oral cavity nebulization was applied with $2 \%$ simple lidocaine for $10 \mathrm{~min}$ (Figure 6-7). At the bottom of both nostrils, they were impregnated with ispes with $2 \%$ simple lidocaine. It was not possible to perform superior laryngeal nerve block due to the distortion of the anatomy. Finally, 10\% simple lidocaine was administered by spray through the oral cavity by two shots.

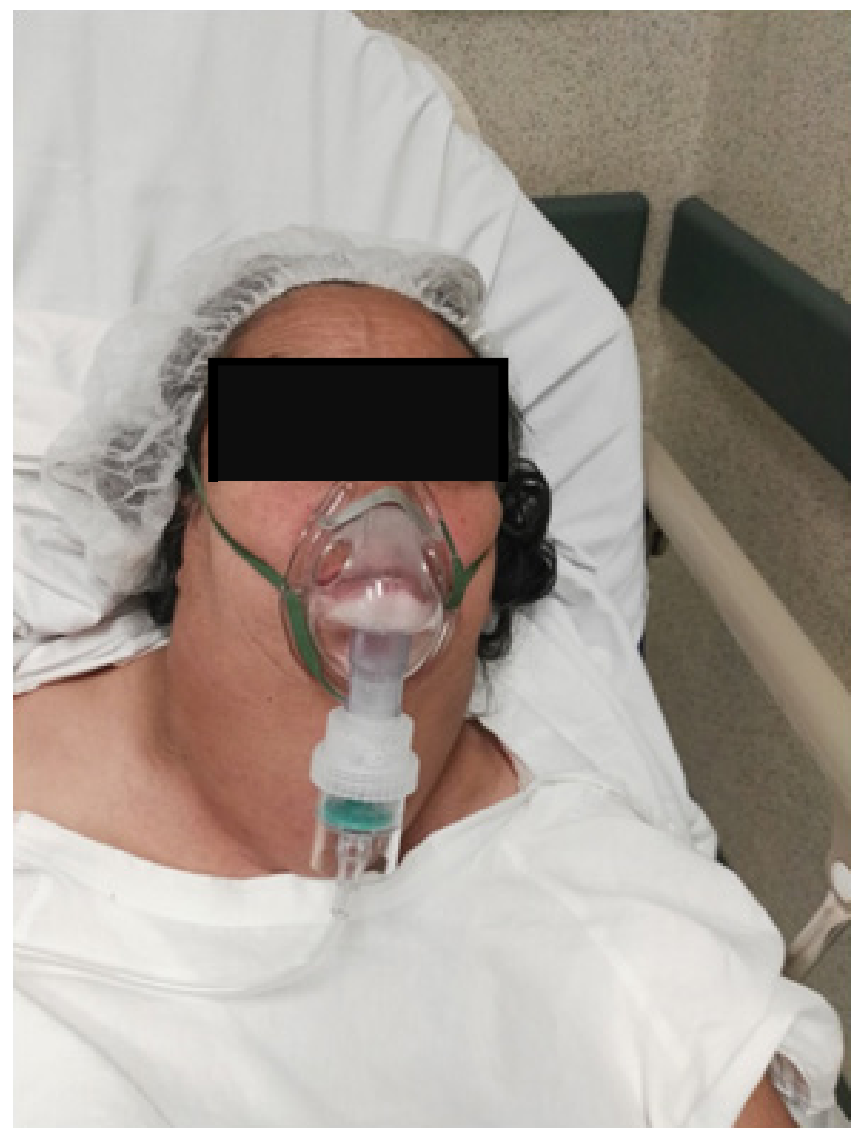

Figure 6

Citation: Barbabosa BMA,Vázquez RP, Cortes AHO, et al.Anesthetic management of anaplastic thyroid carcinoma. J Cancer Prev Curr Res . 20 I9; I0(5): I09-I I3. DOI: $10.15406 /$ jcpcr.2019.10.0040I 


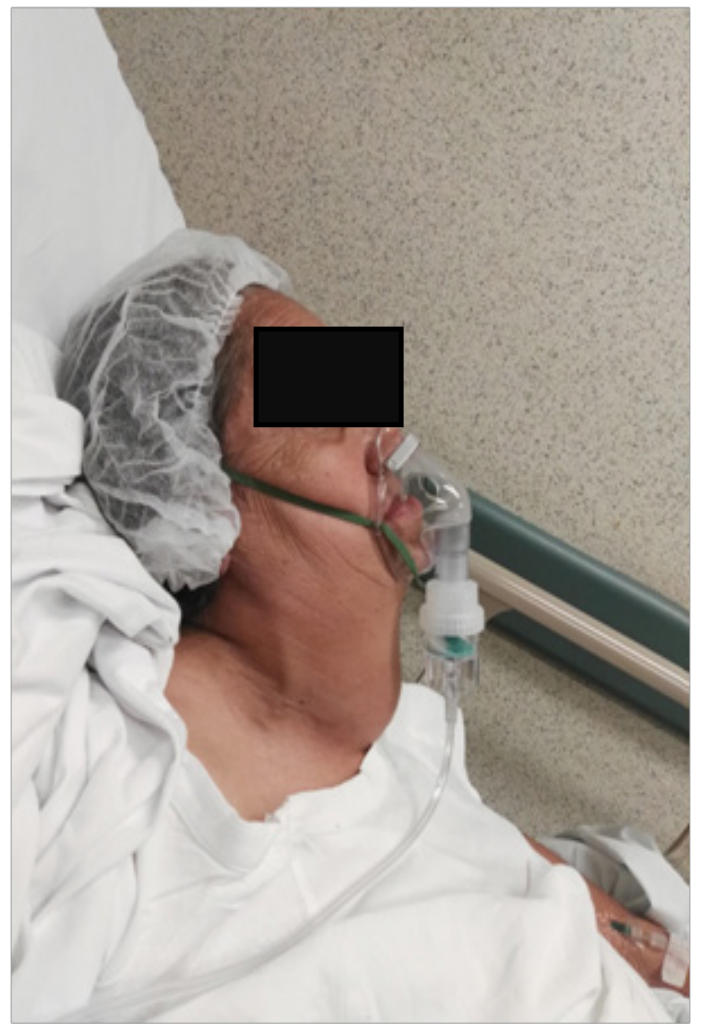

Figure 7

Intravenous fentanyl was administered, after a suitable latency period, the flexible fibroscope was introduced through the oral cavity. The patient maintained spontaneous ventilation and after locating glottis cleared by abundant tumor tissue (Figure 8), simple lidocaine $2 \% 40 \mathrm{mg}$ was installed at the glottis level and once the vocal cords were passed with the fibroscope before the visualization of tracheal rings and tracheal carina, the endotracheal tube of small diameter was introduced.

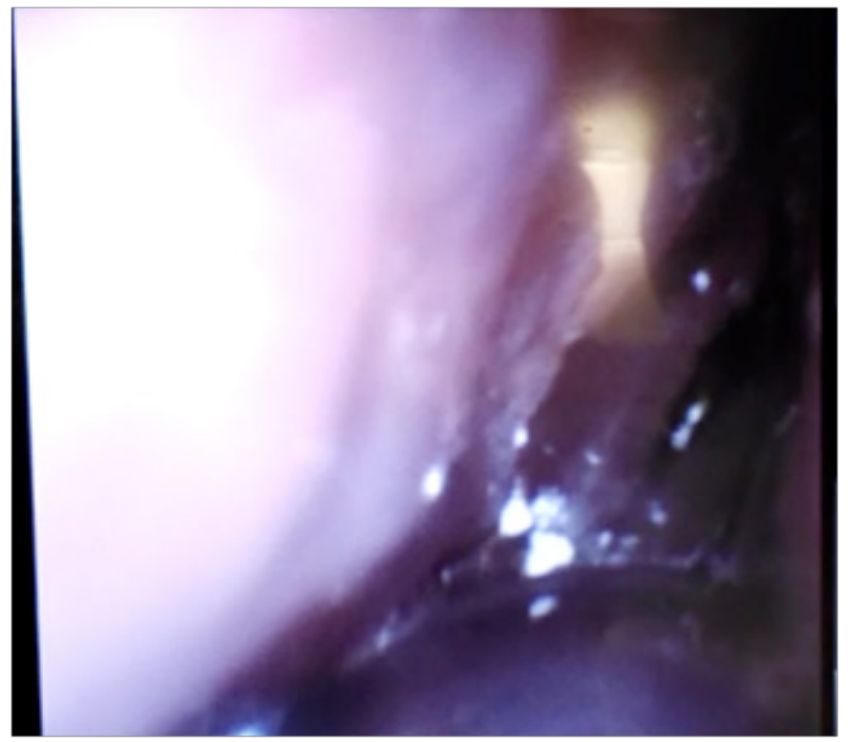

Figure 8
Then, induction is carried out using fentanyl, propofol and vecuronium. Maintenance with an inhaled anesthetic based on Sevoflurane, ventilatory maintenance with low flows, low tidal volume and high respiratory rate (Figure 9-10). At the end of the patient, he leaves with tracheostomy under ventilatory support to schedule withdrawal of ventilatory support (Figure 11), finish surgical procedure without complications. Capnography was normal throughout the procedure. With histopathological result anaplastic carcinoma of the thyroid (Figure 12), and subsequent reference to a center specialized in oncology.

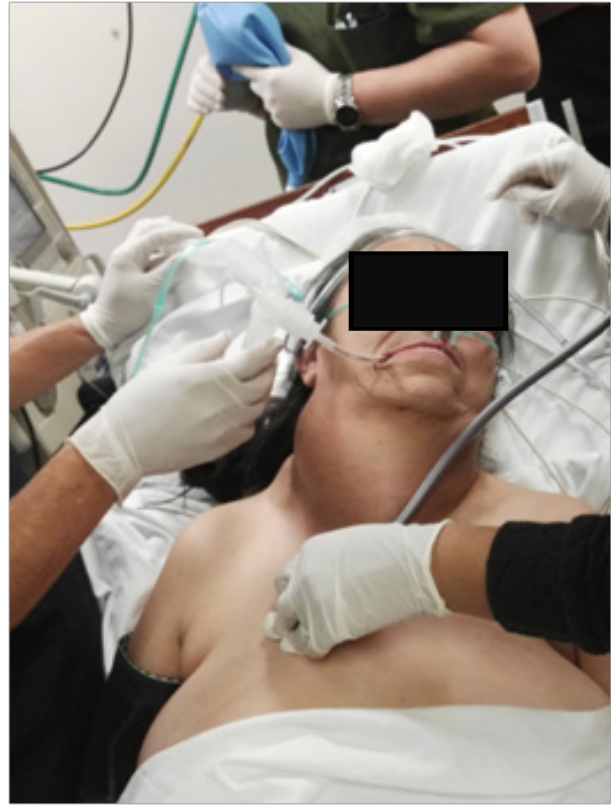

Figure 9

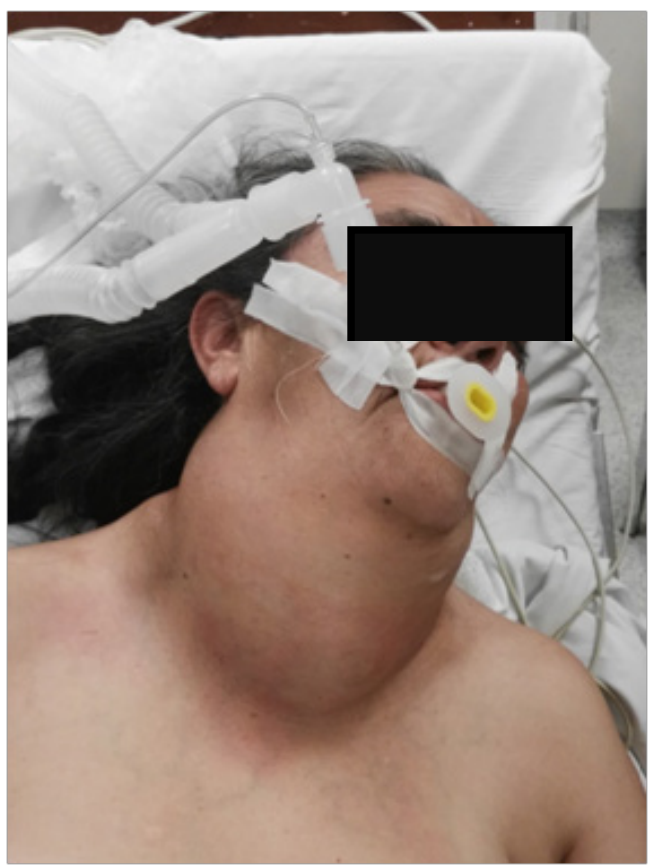

Figure 10 


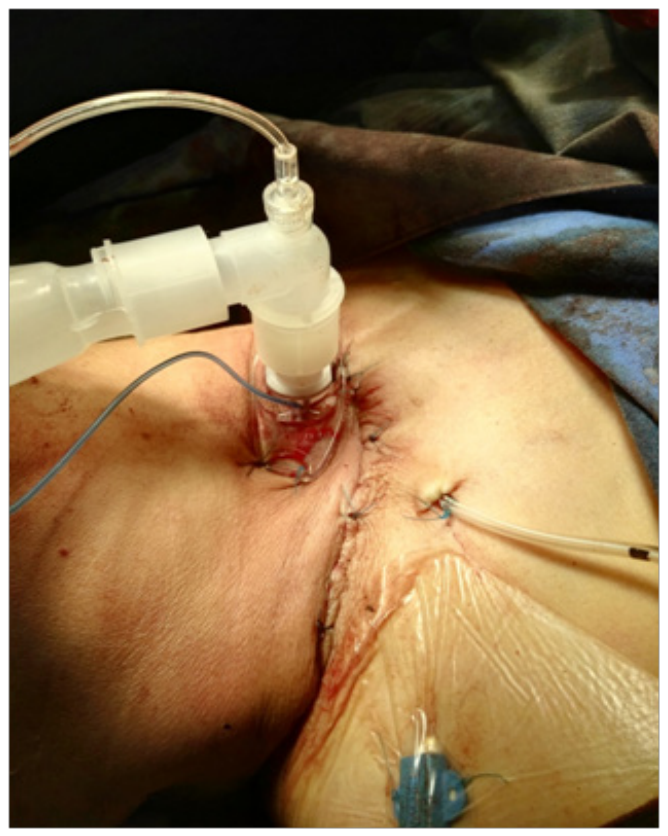

Figure I I

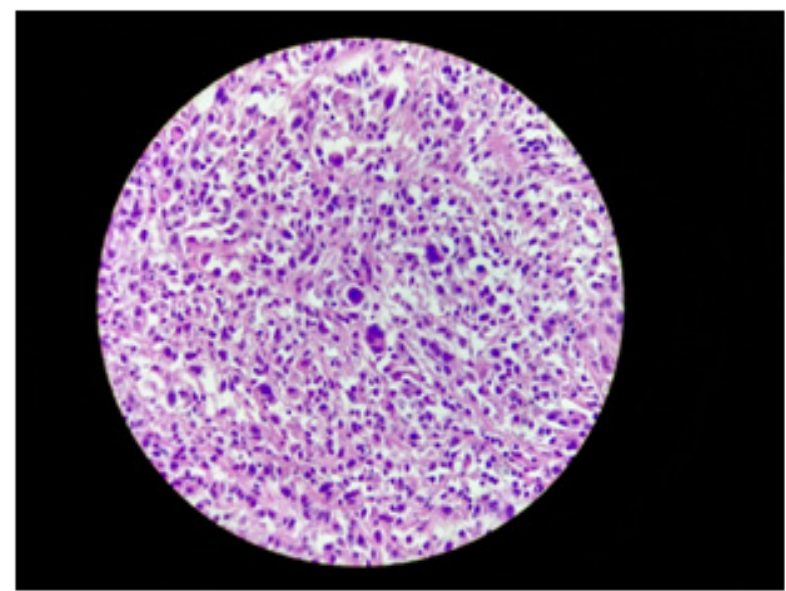

Figure I 2 Anaplastic thyroid carcinoma; High grade pleomorphic tumor cells, neoplastic giant cells, spindle cells or squamoid cells in a background of tumor diathesis and inflammation.

\section{Conclusion}

There are different ways of approaching the airway in goitre cases, as is the case we present. The fibrobronchoscope is the most accepted of them all, either orally or nasally, depending on the opening of the mouth, since a minimum of two centimeters of mouth opening is needed, however it also depends on the experience and skill of the operator, which requires a learning curve, it is not always possible to have a fibrobronchoscope, so an alternative is the Ascope videoscope. Which is much cheaper and requires less experience with its use. ${ }^{16}$

The patient presented goiter. The anesthesiologist's main concern is the potential risk of the difficult airway. It is also important for the surgeon to understand the complementary or contributory role, to help maintain and secure the airway if necessary. It was also decided that in the absence of successful ventilation and intubation, an emerging tracheotomy could be the last resort with a significant technical difficulty due to anatomical alterations.

The Ascope Videoscope has an $80^{\circ}$ field of view $\left(120^{\circ}\right.$ top-down mobility) and an LED lighting method, which allows improved vision. The maximum width of the insertion cord is $5^{\prime} 4 \mathrm{~mm}$, so the minimum size of the endotracheal tube to be used will be six mm ID. The total length of the insertion cord is $630 \mathrm{~mm}$. It has a work channel of 0 ' $8 \mathrm{~mm}$ designed for instillation of local anesthetic through and a luer connector that attaches to this port. The basic principle involved in the process of airway control in a patient with difficult airway is the preservation of spontaneous breathing, with possibilities of vomiting and aspiration.

Goiter caused a potentially difficult VA in a patient under review, limiting mouth opening. Although the longitudinal alignment of the oral, laryngeal and tracheal axes was still possible, this distortion due to the increase in volume limited access to the glottis. The difficulty of the respiratory tract increased the risk of anesthesia with the possibility of hypoxia and increased morbidity and mortality. It is therefore a challenging aspect of anesthesia practice. ${ }^{17}$

The laryngeal mask is also a second safe option, as long as the displacement of the glottis allows it, and its role in the difficult airways is firmly established. Its use in this case was very limited due to anatomical alterations. A video laryngoscope could also be another alternative, as long as the video laryngoscopy sheet enters the oral cavity perfectly well.

\section{Acknowledgments}

None.

\section{Conflicts of interest}

The authors declare there is no conflict of interest.

\section{References}

1. Gharib H, Papini E, Jeffrey R Garber, et al. AACE/AME Task Force on Thyroid 780. Nodules. American Association of Clinical Endocrinologists and Associazione Medici Endocrinology medical guidelines for clinical practice for the diagnosis and management of thyroid nodules. Endocr Pract. 2006;12(1):63-102.

2. Shaha AR. Surgery for benign thyroid disease causing tracheoesophageal compression. Otolaryngol Clin North Am. 1990;23(3):391-401.

3. Thusoo TK, Gupta U, Kochhar K, et al. Upper airway obstruction in patients with goiter studies by flow volume loops and effect of thyroidectomy. World J Surg. 2000;24(12):1570-1572.

4. Ayabe H, Kawahara K, Tagawa Y, et al. Upper airway obstruction from a benign goiter. Surg Today. 1992;22(1):88-90.

5. Hassard AD, Holland JG. Benign thyroid disease and upper airway obstruction: case presentations, pathophysiology, and management. $J$ Otolaryngol. 1982;11(2):77-82.

6. Mack E. Management of patients with substernal goiters. Surg Clin North Am. 1995;75(3):377-394.

7. Raffaelli M, De Crea C, Ronti S, et al. Substernal goiters: incidence, surgical approach and complications in a tertiary referral center. Head Neck. 2011;33(10):1420-1425.

8. de Perrot M, Fadel E, Mercier O, et al. Surgical management of mediastinal goiters: when is a sternotomy required? Thorac Cardiovasc Surg. 2007;55(1):39-43. 
9. Moran JC, Singer JA, Sardi A. Retrosternal goitre: a six year review. Am Surg. 1998;64(9):889-893.

10. Ben Nun A, Soudack M, Best LA. Retrosternal thyroid goiter: 15 years experience. Isr Med Assoc J. 2006;8(2):106-109.

11. Huins CT, Georgalas C, Mehrzad H, et al. A new classification system for retrosternal goiter based on a systematic review of its complications and management. Int J Surg. 2008;6(1):71-76.

12. Findlay JM, Sadler GP, Bridge H, et al. Post-thyroidectomy tracheomalacia: minimal risk despite significant tracheal compression. Br J Anaesth. 2011;106(6):903-906.

13. Rahim AA, Ahmed ME, Hassan MA. Respiratory complications after thyroidectomy and the need for tracheostomy in patients with a large goiter. Br J Surg. 1999;86(1):88-90.
14. Shen WT, Kebebew E, Duh QY, et al. Predictors of airway complications after thyroidectomy for substernal goiter. Arch Surg. 2004;139(6):656660

15. Bensghir M, Bouhabba N, Fjouji S, et al. Difficult intubation: should follow the recommendations. Ann Fr Anesth Reanim. 2014;33(3):181184.

16. Latifi R, Harper J, Rivera R. Total thyroidectomy for giant goiter under local anesthesia and Ketamine in a surgical mission. Int J Surg Case Rep. 2015;8C:52-54.

17. Loftus PA, Ow TJ, Siegel B, et al. Risk factors for perioperative airway difficulty and evaluation of intubation approaches among patients with benign goiter. Ann Otol Rhinol Laryngol. 2014;123(4):279-285. 